\title{
The Development of a Software System for Patients with Breast Cancer Receiving Chemotherapy Treatment: A Pilot Study
}

\author{
Maria Skouroliakou ${ }^{1}$, Ekaterini Vourvouhaki ${ }^{1}$, Ioannis Tsamis ${ }^{2}$, Marianna Sykara ${ }^{2}$, \\ Sofia Kyroudi-Markantoni ${ }^{3}$ and Dimitrios Pappas ${ }^{4}$ \\ ${ }^{1}$ Nutrition and Dietetic Department, Harokopio University, Athens, Greece \\ ²Dietetic Department, “Iaso" Hospital, Athens, Greece \\ ${ }^{3}$ Laboratory of Biopharmaceutics and Pharmacokinetics, School of Pharmacy, University of Athens, \\ Athens, Greece \\ ${ }^{4}$ Department of Statistics, Athens University of Economics and Business, Athens, Greece
}

Correspondence should be addressed to: Maria Skouroliakou; mskour@hua.gr

Received 11 March 2013; Accepted 30 April 2013; Published 25 June 2013

Academic Editor: Maria Evelina Fantacci

Copyright (C) 2012 Maria Skouroliakou, Ekaterini Vourvouhaki, Ioannis Tsamis, Marianna Sykara, Sofia Kyroudi-Markantoni and Dimitrios Pappas. Distributed under Creative Commons CC-BY 3.0

\begin{abstract}
Until recently, anti-cancer clinics in order to plan and schedule chemotherapy treatments applied time-consuming methods, characterized by a high risk of error occurrence. Considering all the barriers that make chemotherapy treatment and demanding procedure, to evaluate if a chemotherapy management program could improve the administration of chemotherapy treatments. The authors developed "Carci", a software tool designed to support anti-cancer clinics. The particular software program aims to provide an improved administration system for injectable anticancer agents and eliminate medication errors that could yield fatal events. The recording of patients' data, the selection of the appropriate treatment protocol, the calculations of drug dosages, the correct preparation and administration of injectable solutions and the updating of secretariat, were conducted both manually and by using the computerized method. These two methods were compared and contrasted. The use of "Carci" resulted in a decrease in the time required to calculate patients' Body Surface Area (BSA) and in a decrease in the occurrence of errors concerning the dosage of medication administered. A total of 18 patients with breast cancer were assigned to this pilot study. Significant differences were observed in BSA values $(p=0.0391)$ and dosage $(p=0.0042)$ between the computerized method "Carci" and manually by the doctor. Although this has been a pilot study, further evaluation of this software program in a larger sample of patients is required. Preliminary data, however, suggests that "Carci" can minimize the risk for error occurrence making chemotherapy treatment faster, easier and more accurate.
\end{abstract}

Keywords: Cancer, chemotherapy treatment, software program, treatment protocols.

Cite this Article as: Maria Skouroliakou, Ekaterini Vourvouhaki, Ioannis Tsamis, Marianna Sykara, Sofia Kyroudi-Markantoni and Dimitrios Pappas (2013), "The Development of a Software System for Patients with Breast Cancer Receiving Chemotherapy Treatment: A Pilot Study," Advances in Cancer Research \& Treatment, Vol. 2013 (2013), Article ID 260996, DOI: 10.5171/2013. 260996 


\section{Introduction}

Chemotherapy is the process of using of chemical agents or medications to treat cancer. It is a systemic therapy that affects the whole body, since the action of chemotherapeutic agents is not limited to malignant tissue, but it also affects normal cells. Administration routes vary, including oral, intravenous, intraperitoneal administration or delivery via catheter. The severity of the side effects commonly depends on the agents used, the dosage, the duration of treatment and treatment cycles, on other accompanying drugs and the individual's response to received medication, as well as the patients' health status. However, it is vital that the agents are administered at a patient-appropriate dosage in order to yield optimal results and prevent adverse events due to toxicities ${ }^{1}$. Dosage individualization is of significant importance, since it prevents toxicity due to overdose and avoids treatment failure due to inadequate drug administration ${ }^{2,3}$. The guidelines for the safe handling and the use of anticancer agents have been established by the National Institute of Health and the American Society of Hospital Pharmacists 4 .

Hospitals and clinics providing chemotherapy treatments face a number of problems on daily routine tasks, such as updating patients' records and operational problems concerning the overall organization of treatments and medication management. The time spent on defining the appropriate treatment protocols, calculating drug dosages and specifying their preparation instructions is usually long, and cuts down on health care professionals' productivity. Also, professionals from different departments who need to cooperate in order to provide optimal treatment, they usually lack immediate access to important information waiting for data to be collected from each department. Additionally, work overload does not allow doctors to be constantly up to date concerning new medications and chemotherapeutic agents, which may be improved or may be less expensive than old, outdated ones. An initial attempt was made by Okayasu et al. in 2009 in order to minimize errors on the preparation of chemotherapy treatments by developing a computer-assisted biohazard safety cabinet enabling for the safe handling and preparation of the mixture ${ }^{5}$.

\section{Aim of the Study}

The purpose of the present pilot study is to present and describe "Carci", a software tool developed to meet the needs of anticancer clinics in improving the chemotherapy treatment procedures and in decreasing their operational cost.

\section{Method}

\section{Technical Details of the Program}

The software was developed in Visual $\mathrm{C}^{++}$ using Microsoft Visual Studio 2010. Minimum operating system requirements include Microsoft Windows XP, service pack 2. It is mandatory that each computer can be connected with an Ethernet network, wired or wireless. There are no requirements concerning the processor, but the computer must have at least $1 \mathrm{~GB}$ of RAM. To use the program in a system network, there must be a Server, for which there are no specific requirements, but the ability to install an SQL database. The network is recommended to be local, not allowing Internet access, in order to secure confidentiality.

\section{Study Population}

Data were recorded from 18 individuals, 17 females and 1 male. Recruitment took place at IASO Hospital of Athens, during a 6month period. All patients were diagnosed with breast cancer.

The study was approved by the ethical committee of the hospital and conducted in compliance with the ethical principle of the Helsinki Declaration. All information regarding participants' clinical condition, medication and medical history were provided by the participants during their interview with the clinicians. Documents regarding medical tests and biochemical data were collected. Patients' characteristics are presented in Table 1. 
Table 1: Demographic and Medical Characteristics of the Study Patients

\begin{tabular}{|lc|}
\hline Characteristics & $\mathbf{n}$ \\
\hline Sex $($ male/Female $)$ & $1 / 17$ \\
\hline Age $($ years $)$ & $54.72 \pm 12.34$ \\
\hline BMI $\left(\mathrm{kg} / \mathrm{m}^{2}\right)$ & $29.4 \pm 5.8$ \\
\hline
\end{tabular}

\section{Statistical Analysis}

In order to investigate any potential difference between the therapeutic choices of the Doctors in charge and the automatic choices of the "Carci" software used, we performed a Wilcoxon non-parametric rank test for paired samples ${ }^{6}$, due to the fact that our data did not meet the normal distribution criteria based on a Jarque-Bera hypothesis test ${ }^{7}$. A p-value of $<0.05$ was considered significant for all our statistical analyses. All calculations have been performed using Matlab $^{\circledR}$ R2007a Technical Software ${ }^{8}$.

\section{Results}

The support system "Carci" is comprised of two subsystems, "CarciMed" and "CarciWard". "CarciMed" is the drug database and it is a separate entity to the patient database "CarciWard". "CarciMed" provides the interface to set and configure the protocols and drugs available to the system. A system vendor called "the administrator" has access to "CarciMed" and is responsible for updating the database on a monthly basis. The administrator can alter and monitor all data with regard to the various treatment protocols, medications, method of administration, sequence and duration of administration of the chemotherapeutic agents.

Currently, only breast cancer chemotherapy treatments are supported, therefore only medicines and protocols relevant to that aim have been configured. More specifically, there are 19 protocols for complementary chemotherapy (adjuvant) and 5 for locally extended breast cancer (locally advanced treatment), according to the international guidelines ${ }^{9}$. The injectable chemotherapeutic agents that are present in these protocols and consequently exist in the database are $10^{10,11}$.
Biochemical factors and additional pathological conditions that may influence the dosage of administration of every chemotherapeutic agent are also taken into account in the "CarciMed" database. Additionally, "CarciMed" holds all relevant information regarding the recommended infusion method (IV push, IV infusion) and the compatibility of each solvent with each anticancer agent.

"CarciMed" is linked with "CarciWard", the platform developed to support anti-cancer clinics. "CarciWard" provides a number of forms with patient-related information and each of them can be filled in by the Health Care Professionals within the hospital or the clinic. The clinic secretary is entitled to have access, make additions, deletions or modifications only in the form "PATIENT INFORMATION". Doctors have access in all forms, apart from the one with "ADMINISTRATOR'S INFORMATION". More specifically, they may view and intervene in the forms "PATIENT INFORMATION", "DIAGNOSTIC INFORMATION", "MEDICAL HISTORY" and "THERAPY PROTOCOL". Nurses have access in two forms, "PATIENT INFORMATION" and "MEDICAL HISTORY". Pharmacists have access in the form "THERAPY PROTOCOL" only, to which they cannot intervene. Health Care professionals from different departments are entitled to have selected access. The reasons behind the different access levels granted to the Health Care professionals are two-fold; the fact that unauthorized staff intervenes to patients' data or treatments may be very dangerous for a patient's health, since even the minor modification in a drug dosage may be fatal. Thus, access in the program is always named protocol, and dosage modifications always require doctor's approval. The second reason is that the personal details of the patients should be kept confidential. This is accomplished by restricting view access, though, according to the work force credentials that are used; 
the needed information for the accomplishment of the needed work tasks is provided.

"CarciWard" is comprised of five electronic forms; each of them has several subsections and fields to be filled in. Health Care Professionals have selected access to one or more of the forms, depending on their specialty. The "CarciWard" software administrator, which is usually the Head of the Clinic, has access to all of them. All, doctors, nurses, pharmacists, secretaries and the administrators may access the forms they are entitled to, searching for the information they need either by the date of a patient's treatment or by the patient's name.

The form "PATIENT INFORMATION" contains the patient's personal information (name, clinic, doctor in charge, insurance fund), contact information, non-cancer pharmaceutical treatment, allergies, date of birth, sex, height, weight, Body Surface Area (BSA) and appointment date (figure 1). The system has also a specific algorithm in order to automatically calculate the BSA of the patient, of each person, a factor that highly affects the dosage of the chemotherapeutic agents. The level of BSA is calculated based on the values given for height and weight ${ }^{12}$. Patient's personal information may be viewed once a patient's name has been selected from the list that appears at the upper part of the form. Access to this form is permitted to clinic Health Care Professionals (doctors, pharmacists, nurses) and the clinic secretary.

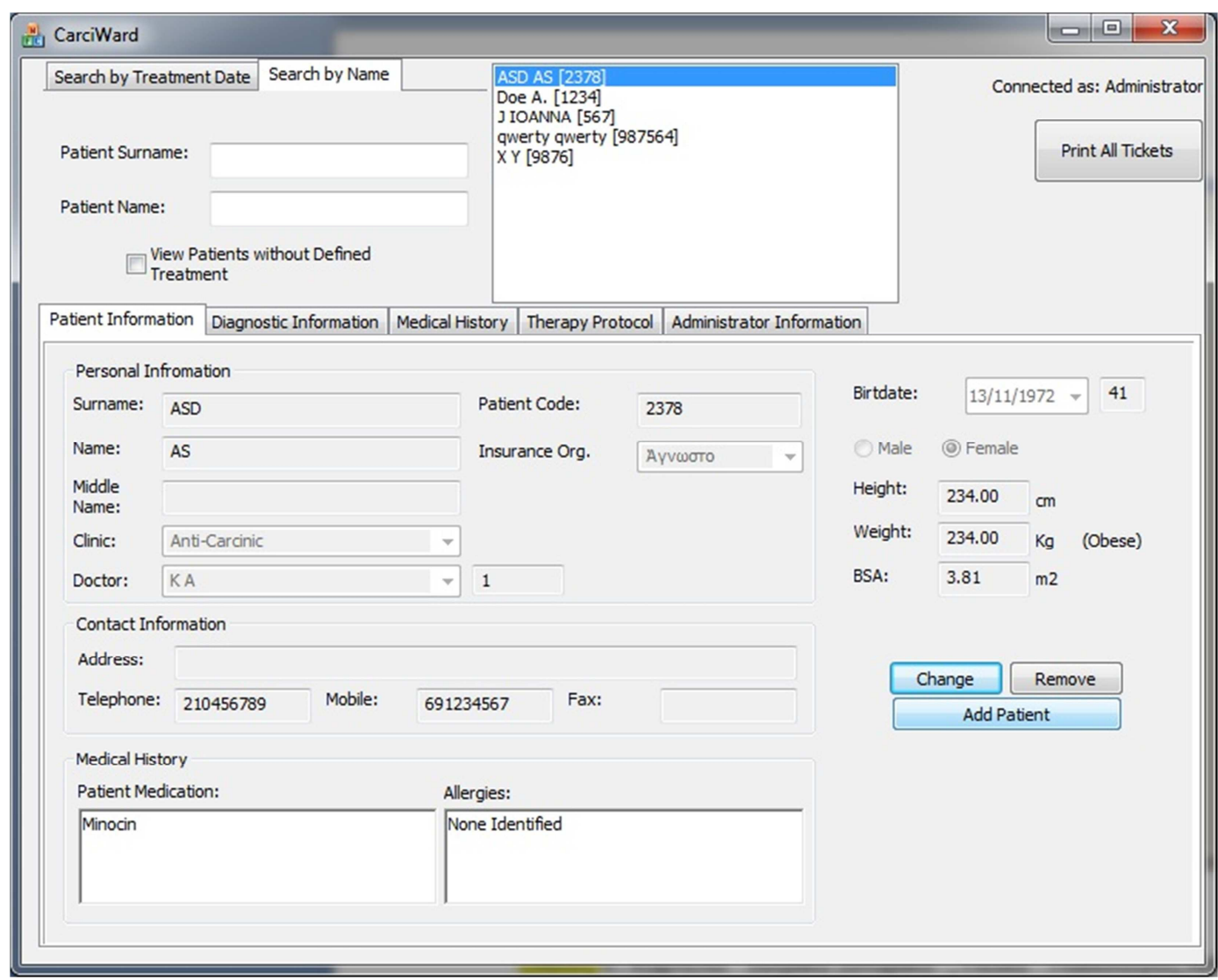

Figure 1: Patient Information form on CarciWard. In this Form, the Patient's General Information is Recorded (Name, Surname, Date of Birth, Contact Information) Including also the Height, Weight and BSA of the Patient. 
The form "DIAGNOSTIC INFORMATION" contains information about the status and the stage of cancer of the patient (figure 2). The doctor completes information about whether the patient has undergone surgery or lymph node dissection (in cases of breast cancer), defines the clinical stage of cancer, the multiplication ratio and gives a final diagnosis. The doctor also defines the treatment protocol. In addition, old protocols appear in the form, so that the doctor can access the treatment record of a patient.

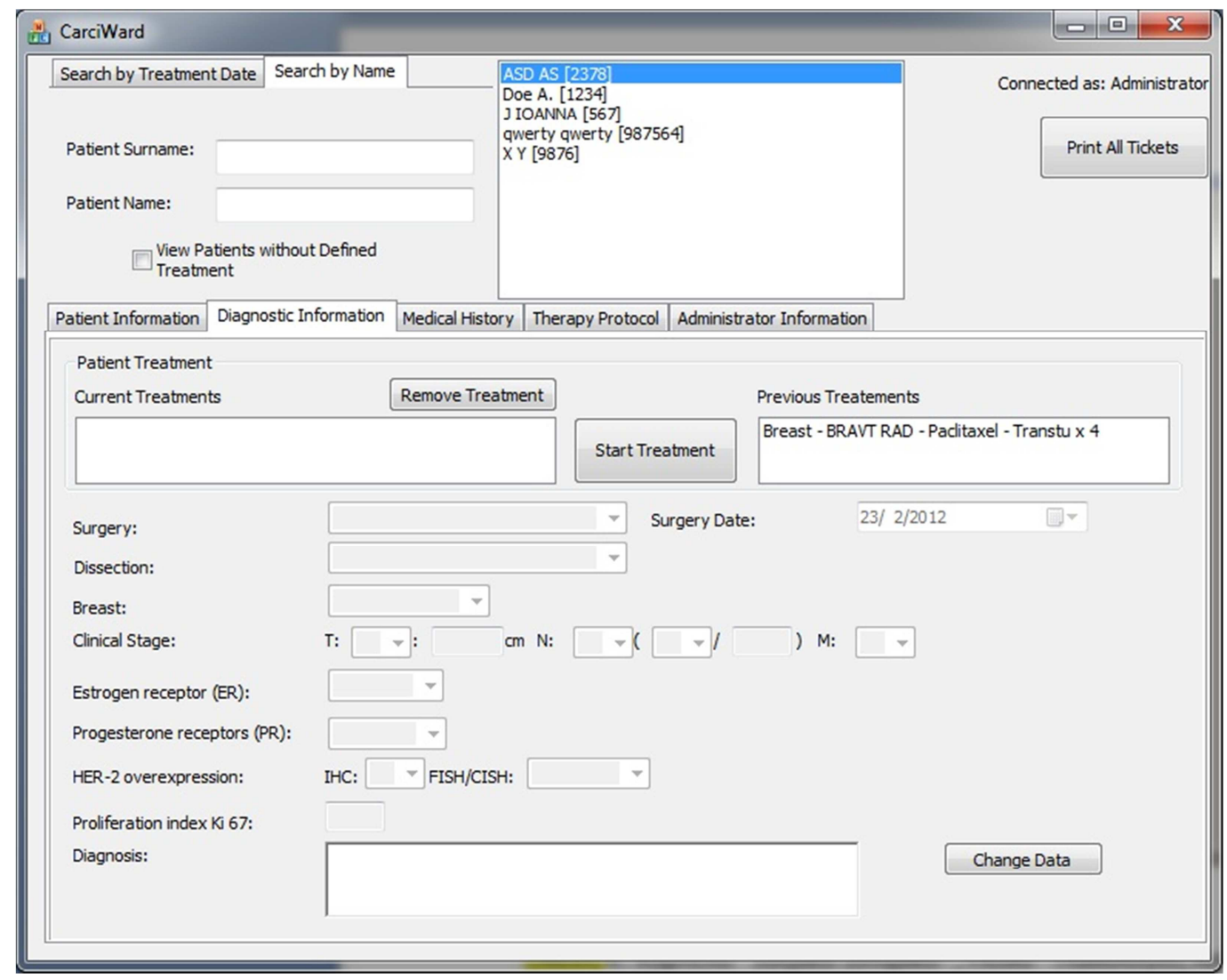

Figure 2: Diagnostic Information form on CarciWard. Information on Previous Surgeries, Dissection, Clinical Stage of the Patient, Estrogen and Progesterone Receptor and HER-2 Overexpression Values are Recorded.

The form "MEDICAL HISTORY" contains data on lab test results of the patient (figure 3). 


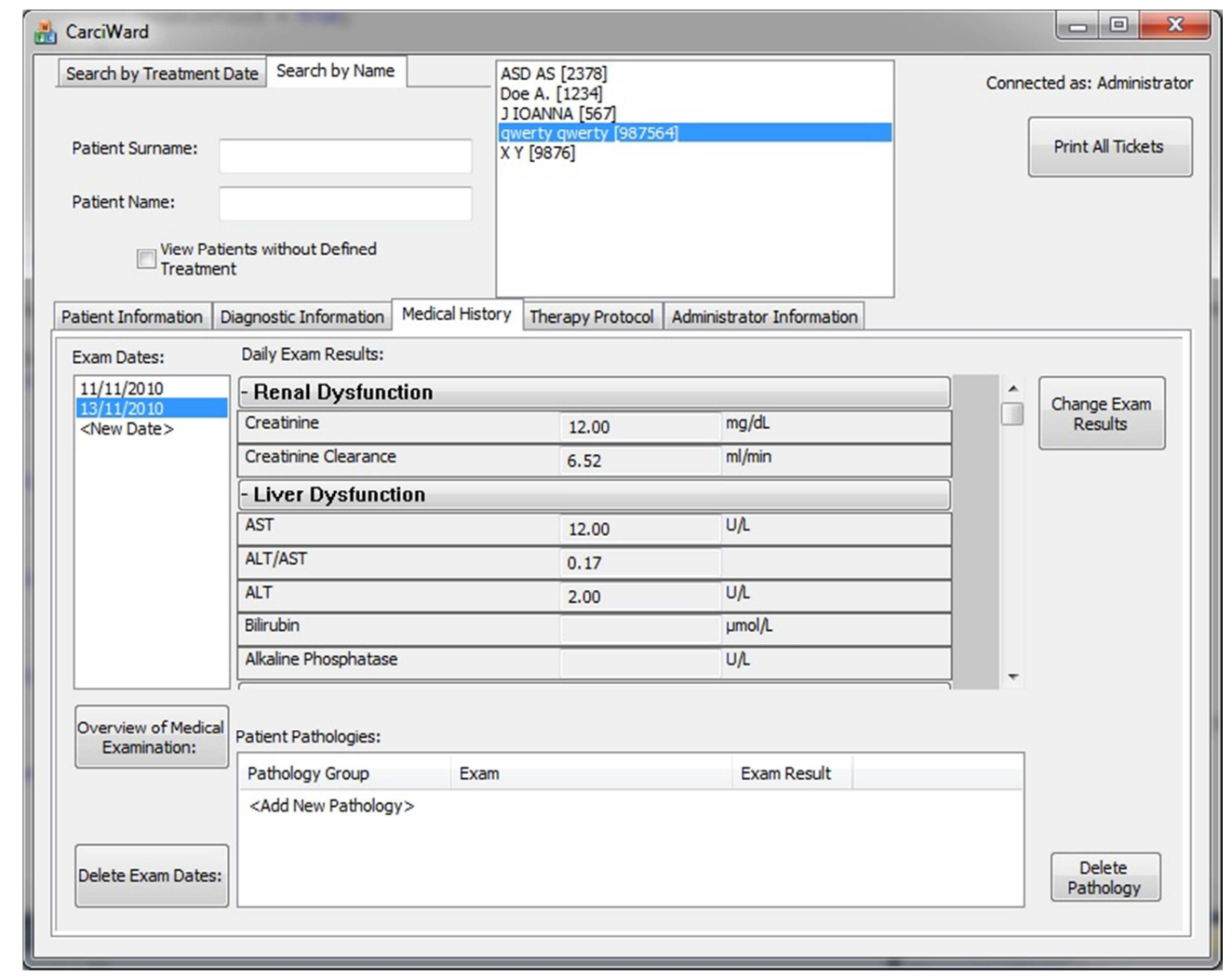

Figure 3: Medical History form on CarciWard. The Medical History of the Patient is Recorded on this Form, Such as the Degree of Renal and Liver Dysfunction Estimated through Various Factors on Blood Test

Test date is typed and lab test results such as kidney, liver and cardiac function, platelet and white blood cell count, serve as input data (Table 2).

Table 2: Shows the Biochemical Factors Needed to be Recorded on the "MEDICAL HISTORY" Form.

\begin{tabular}{|ccc|}
\hline TEST & $\begin{array}{c}\text { BIOCHEMICAL FACTOR } \\
\text { MEASURED }\end{array}$ & MEASUREMENT UNIT \\
\hline Renal Dysfunction Degree & Creatinine, Cr* & $\mathrm{mg} / \mathrm{dL}$ \\
\hline Renal Dysfunction Degree & Creatinine Clearance, ClCr & $\mathrm{mL} / \mathrm{min}$ \\
\hline Liver Dysfunction Degree & AST & $\mathrm{U} / \mathrm{L}$ \\
\hline Liver Dysfunction Degree & ALT/AST & $\mathrm{U} / \mathrm{L}$ \\
\hline Liver Dysfunction Degree & ALT & $\mu \mathrm{mol} / \mathrm{L}$ \\
\hline Liver Dysfunction Degree & Bilirubin & $\mathrm{U} / \mathrm{L}$ \\
\hline Liver Dysfunction Degree & Alkaline Phosphatase & $\mathrm{x} 10^{9} / \mathrm{L}$ \\
\hline Blood Count & Platelets, PLT & $\mathrm{x} 10^{9} / \mathrm{L}$ \\
\hline Blood Count & Neutrophils, ANC & $\%$ \\
\hline Cardiac Dysfunction & Left Ventricular Ejection & Fraction, LVEF \\
\hline
\end{tabular}


In case where the lab test results indicate the presence of a co-existing clinical/pathological condition, the doctor needs to add the condition in the field "Patient's Pathologies". As pathologies, there have been listed Pathological Conditions/ Manifestations or/and Tests that take place during chemotherapy treatment and cannot be measured through a daily number value.

The pathologies that have been studied for this program and commonly alter the dosage of injectable chemotherapy agents are the following:

Blood Pathologies: Febrile Neutropenia, Intolerable Bone Pain
Neuropathies: Neurotoxicity, Peripheral Neuropathy

Renal Dysfunction: Dialysis, Continuous Ambulatory Peritoneal Dialysis (CAPD)

Common Clinical Manifestations: Joint Pain and/or Muscle Pain, Abnormal Fluid Retention, Third-Space Fluid

\section{Body Characteristics: Obesity}

In the form "THERAPY PROTOCOL", the doctor and the pharmacist may view the most recent treatment protocol, as well as older ones, searching them by the date of treatment (figure 4).

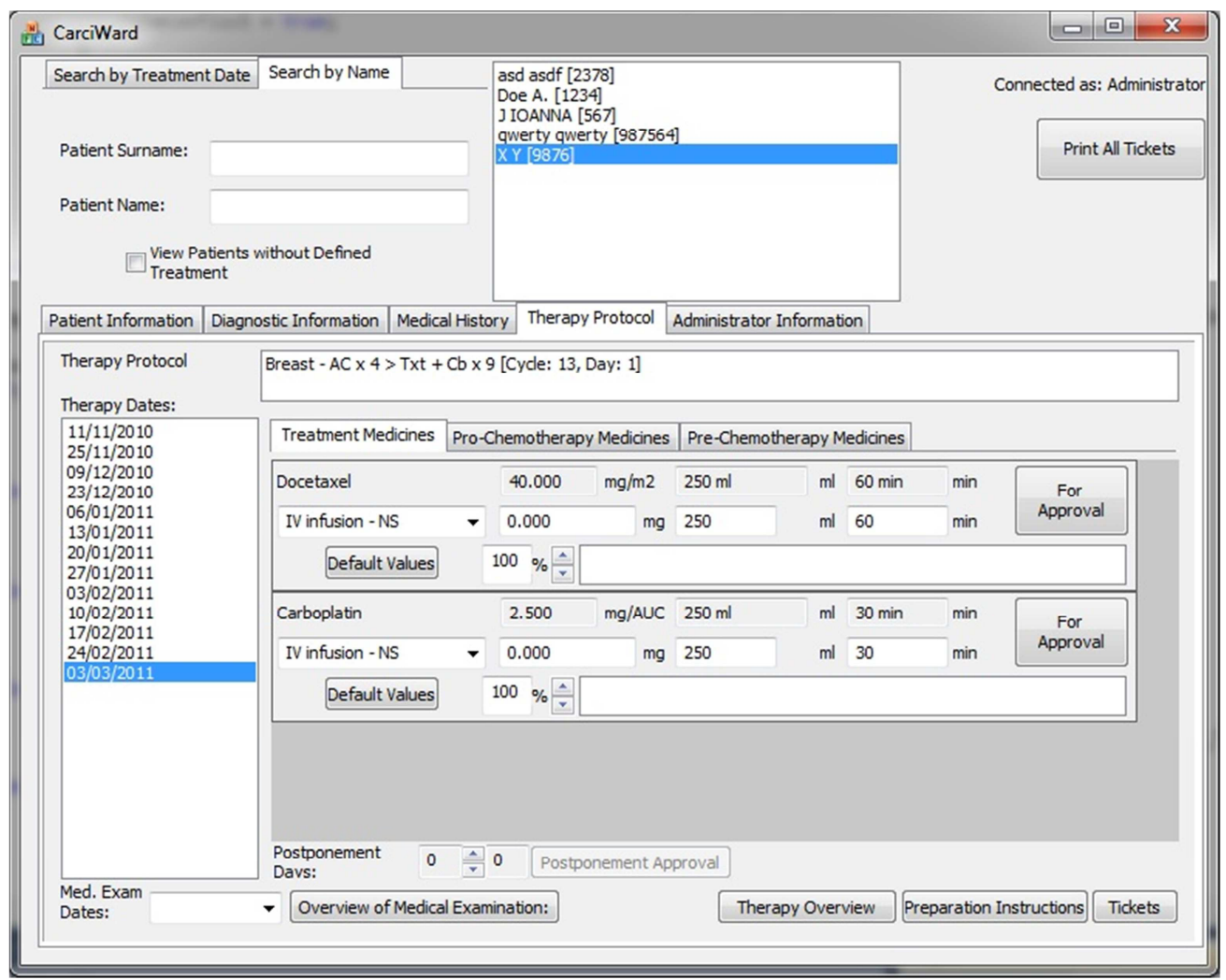

Figure 4: Therapy Protocol form on CarciWard. In this Form, there is a List of Drugs, the Defined Dosage and Administration Rate, the Manner of Infusion (IV Push, IV Infusion) Depending on the Injectable Chemotherapeutic Agent, the Chemotherapy Protocols, the Solvent Compatible with Each Anticancer Agent, the Approved Volume of the Infusion Solvent and the Infusion Rate of the Final Solution.

There are also three subsections, presenting current "Treatment Medicines", "Pre-Chemotherapy Medicines" and "PostChemotherapy Medicines". Under those sections there are lists with drugs (listed by generic name), the defined dosage and administration rate, the manner of infusion (IV push, IV infusion) depending on the 
injectable chemotherapeutic agent, the chemotherapy protocols, the solvent compatible with each anticancer agent, the approved volume of the infusion solvent and the infusion rate of the final solution. The program suggests a particular dose and administration rate for a patient, calculated automatically based on patient's BSA. The doctor may intervene and alter the dose and the administration rate, if that is considered necessary. He/she may also view lab tests by selecting the appropriate key, and modify doses or administration rate based on test results. At this stage, the program sets questions concerning patient's biochemical values. The answers the user gives add up and then it appears a final order concerning the alterations that need to take place on the treatment schema. The pharmacist is entitled to read preparation instructions and print the labels that will be stuck on the vessels carrying the solutions.

The form “ADMINISTRATOR'S INFORMATION" contains any kind of information the administrator may need to use in order to add or alter fields and data in the software program. If for example, an outdated chemotherapy agent needs to be substituted with a new one, the administrator may store the new information in this form, in order to incorporate it in "CarciMed" later, or vice versa.

Using a Jarque-Bera hypothesis test we observed that the two datasets did not follow the normal distribution for the BSA, dosage, and time values. We therefore performed a Wilcoxon non-parametric rank test for statistical comparison between paired samples. A p value of less than 0.05 was regarded as statistically significant. Significant difference was observed in BSA values $(p=0.0391)$ and dosage $(p=0.0042)$ between "Carci" and doctor. However, no significant difference was observed for the time of injection $(\mathrm{p}=0.4814)$ between "Carci" and doctor.

Table 3: Median Values for BSA, Dosage and Time Compared between "Carci" and the Manual Preparation by the Doctor.

\begin{tabular}{|l|c|c|c|}
\hline Table 3. & \multicolumn{4}{|c|}{ Median Values } \\
\hline Variable & CarciWard & Doctor & p-value \\
\hline BSA $\left(\mathrm{m}^{2}\right)$ & 1.82 & 1.8 & 0.0391 \\
\hline Dosage $(\mathrm{mg})$ & 857.33 & 200 & 0.0042 \\
\hline Time $(\mathrm{min})$ & 40 & 60 & 0.4814 \\
\hline
\end{tabular}

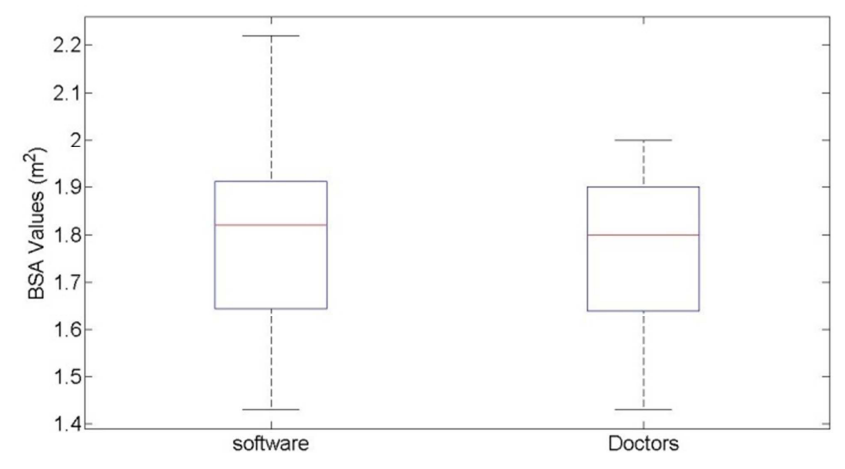

Figure 5: Boxplot of the BSA Values, Carci vs Doctors, with the Median in Red. 


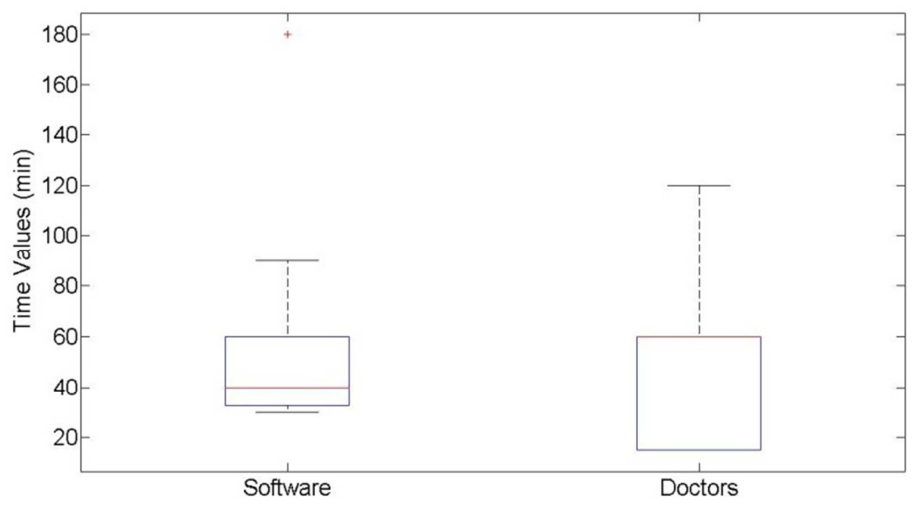

Figure 6: Boxplot of the Time Values, Carci vs Doctors, with the Median in Red.

\section{Discussion}

Excellent organizational skills, knowledge competence and professional responsibility are three major characteristics that clinical staff needs to have. All members of the clinical staff need to know what their exact duties are in order to complete their tasks fast, accurately and without being destructed from information and data that are unnecessary to them.

"Carci" has been developed to facilitate the work of hospital and clinic professionals, by providing all those data and information needed to carry out the daily work. The fact that a nurse does not have access in the same forms as the doctor prevents the first from getting confused and wasting time with unnecessary information, while it authorizes the second to grant data security and confidentiality. Work is done faster and in a more responsible manner, since each one who enters the program, has named access only. Thus, the name of the person who adds, deletes or modifies data, may be viewed at any moment by the colleagues. This means that information and decisions made on protocol selection or modification, drug doses, drug replacements and administration procedures are all name-signed.

The program also functions as a database for treatment protocols and prescribed medication. "CarciWard" administrator, who has access to all 5 forms of the program, is entitled to add, delete or modify protocol and medication related information, as long as new and improved agents are launched in the market. The database may be constantly updated by the system vendor and thus, doctors get informed about new anticancer agents and other related drugs, without making any effort to search for new products. It is virtually impossible that all doctors are aware of all anti-cancer medications that exist in the market, so a database providing this information as well as drug combinations (protocols) is really valuable for them.

The prevention of error occurrence is another advantage of the particular program. Using a manual method to calculate BSA and medication doses, there is an increased risk in error making. However, errors in dose's measurements can be fatal. Our study was the first prospective study to demonstrate the application of CarciWard in clinic. Despite the relative small sample of patients, a significant difference $(p=0.0042)$ in the calculated dosage of the chemotherapeutic agents was found between the CarciWard and that prescribed by the doctor. This reflects the need for a precise protocol that it will eliminate the possibility of an occasionally fatal event. CarciWard produces a detailed protocol for the preparation of the chemotherapy treatment, describing the procedure step by step and giving the precise amounts for each. Apart from miscalculations, errors may also occur when writing or typing the 
name of a medicine. Having a database where a doctor can select a specific chemotherapeutic agent or other supportive medications reduce the possibilities for the occurrence of errors ${ }^{13-}$ 19.

Apart from an anti-cancer protocol and medication database, the program serves as a patients' record, where all patientrelated information is stored and can be retrieved even years later, such as in cases of relapse. Paper and folder filling are not necessary, since all patient information may be stored in the program. Patient data and information can be used for statistical analysis and for research purposes, by the clinics of pharmaceutical companies.

"Carci" is designed initially for use in local facilities. In cases where a patient needs to move to a different clinic, data can be easily exported and imported within the "Carci" system. Access on patient's data through internet will be promoted once secure settings are implemented on the system given the degree of confidential data kept on the system.

\section{Conclusion}

In summary, "Carci" is a software tool that enables us to make fast and precise preparations of injectable anticancer agent mixtures. Although this has been a pilot study, "Carci" may be a promising software tool for the safe management of medication in cancer chemotherapy.

\section{Conflicts of Interest}

The authors have no conflict of interest relevant to the subject of this article.

\section{Acknowledgments}

The authors would like to thank Sofia Papageorgopoulou and Pano Papandreou for their invaluable help during the conduction of this study.

\section{References}

1. Venturini, M., Del Mastro, L., Aitini, E., Baldini, E., Caroti, C., Contu, A., Testore, F., Brema, F., Pronzato, P., Cavazzini, G., Sertoli, M. R., Canavese, G., Rosso, R. \& Bruzzi, P. (2005). "Dose-Dense Adjuvant Chemotherapy in Early Breast Cancer Patients: Results from a Randomized Trial," Journal of National Cancer Institute., 97(23): 1724-33.

2. American Society of Hospital Pharmacy (1990). 'ASHP Technical Assistance Bulletin on Handling Cytotoxic and Hazardous Drugs,' 1990, 47(5): 1033-49.

3. Trinkle, R. \& Wu, J. K. (1996). "Errors Involving Pediatric Patients Receiving Chemotherapy: A Literature Review," Medical and Pediatric Oncology, 26(5): 34451.

4. 'Recommendation for the Safe Handling of Parenteral Antineoplastic Drugs,' Dept of Health and Human Services Publication (NIH) 83-2621. For sale by the Superintendent of Documents, U.S. Government Printing Office, Washington, DC 20402.

5. Okayasu, S., Nakamura, M., Sugiyama, T., Chigusa, K., Sakurai, K., Matsuura, K., Yamamoto, M., Kinosada, Y. \& Itoh, Y. (2009). "Development of ComputerAssisted Biohazard Safety Cabinet for Preparation and Verification of Injectable Anticancer Agents," Chemotherapy, 55(4): 234-40.

6. Hollander, M. \& Wolfe, D. A. (1999). "Nonparametric Statistical Methods," Hoboken, NJ: John Wiley \& Sons, Inc., 1999.

7. Jarque, C. M. \& Bera, A. K. (1987). “A Test for Normality of Observations and Regression Residuals," International Statistical Review. Vol. 55, No. 2, 1987, pp. 163-172. 
8. Attaway, S. (2012). Matlab: A Practical Introduction to Programming and Problem Solving, Elsevier, 2012.

9. www.bccancer.bc.ca/HPI/Chemotherap yProtocols/ Adjuvant chemotherapy

10. www.bccancer.bc.ca/HPI/Chemothera pyProtocols/ Locally advanced treatment.

11. www.bccancer.bc.ca/HPI/DrugDataba se/

12. Dominic, A. Solimando, Jr., B. S. \& Pharm., M. A. (2008). Drug Information Handbook for Oncology, Lexi-Comp, Inc. Canada (7th edition): Alphabetical listing of drugs.

13. Markantonis- Kyroudi .(2000).'Clinical Pharmacokinetics,' 2000, pp 83.

14. Schiff, G. D. \& Rucker, T. D. (1998). "Computerized Prescribing: Building the Electronic Infrastructure for Better Medication Usage," JAMA The Journal of the American Medical Association. 279(13): 1024-9.

15. Shane, R. (2002). "Computerized Physician Order Entry: Challenges and Opportunities," American journal of healthsystem pharmacy. 59: 286-88.

16. Lipton, H. L., Miller, R. H. \& Wimbush, J. J. (2003). "Electronic Prescribing: Ready for Prime Time?," Journal of Healthcare Information Management, 17(4): 72-9.

17. Bell, D. S., Cretin, S., Marken, R. S. \& Landman, B. (2004). "A Conceptual Framework for Evaluating Outpatient Electronic Prescribing Systems Based on Their Functional Capabilities," Journal of the American Medical Informatics Association, 11(1): 60-70.

18. Teich, J. M., Osheroff, J. A., Pifer, E. A., Sittig, D. F., Jenders, R. A. \& Panel, T. C. (2005). "Clinical Decision Support in Electronic Prescribing: Recommendations and an Action Plan," Journal of the American Medical Informatics Association, 12(4): 365-76.
19. Skouroliakou, M., Konstantinou, D., Papasarantopoulos, P. \& Matthaiou, C. (2005). "Computer Assisted Total Parenteral Nutrision for Pre-Term and Sick Term Neonates," Pharmacy World \& Science, 27(4): 305-10.

20. Tamblyn, R., Huana, A., Kawasumi, Y., Bartlett, G., Grad, R., Jacques, A., Dawes, M., Abrahamowicz, M., Perreault, R., Taylor, L., Winslade, N., Poissant, L. \& Pionsonneault, A. (2006). "The Development and Evaluation of an Integrated Electronic Prescribing and Drug Management System for Primary Care," Journal of the American Medical Informatics Association, 13(2): 148159.

21. Shah, N. R., Seger, A. C., Seger, D. L. , Fiskio, J. M. , Kuperman, G. J. , Blumenfeld, B. , Reckler, E. G., Bates, D. W. \& Gandhi, T. K. (2006). "Improving Acceptance of Computerized Prescribing Alerts in Ambulatory Care," Journal of the American Medical Informatics Association, 13(1): 511. 